Journal of Al Azhar University Engineering Sector

Vol. 12, No. 45, October, 2017, 1401-1407

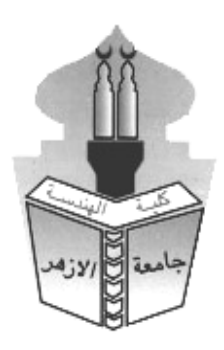

\title{
MA-LEACH: ENERGY EFFICIENT ROUTING PROTOCOL FOR WSNS USING PARTICLE SWARM OPTIMIZATION AND MOBILE AGGREGATOR
}

\begin{abstract} Al-Azhar University, Nasr City, Cairo, Egypt

Routing protocols for wireless sensor networks pay a great attention to the limited resources of the nodes. In this paper, we study LEACH routing protocol and its performance and propose a new protocol, MA-LEACH. We introduce a mobile aggregator which is a gadget adopted to mitigate the overhead on the cluster heads $(\mathrm{CHs})$. In addition, we optimize the trajectory using particle swarm optimization (PSO). Hence, we adapt the TSP problem to our protocol to determine the optimal trajectory that a mobile aggregator could travel to visit every cluster head in the network. We simulate the proposed protocol in MATLAB and the results reveal that it outperforms LEACH in network lifetime and energy consumption. Also, we compare our findings with a recent extension to the LEACH called LEACH with fuzzy descriptors. The simulation results show that MA-LEACH surpasses LEACH with fuzzy descriptor.
\end{abstract}

Alzahraa Elsayed, Mohamed Sharaf and Fawzy Elrefaai

Department of Computer Systems and Engineering,

\section{INTRODUCTION}

A Wireless Sensor Network (WSN) is a collection of resource limited sensor nodes deployed in large numbers to monitor a phenomenon of interest [6]. Since the nodes are energy constrained, the battery lifetime is a major concern. Therefore, energy consumption is an important criterion for the designing of this kind of networks [10]. We apply WSNs in a variety of applications: agricultural, medical, environmental, etc. Major advantages of WSNs are the ability to cover harsh terrains, reliability, accuracy and nevertheless at a possibly lower cost. A great body of research has discussed the benefits of WSNs [8,21].

The purpose of deploying sensor nodes is to gather data form the site of interest. To meet this goal, the nodes need to have the sensing and the communicating capabilities. After the gathering process, the nodes eventually forward the collected data to the base station (BS), a mostly fixed capable device that can receive the data from the nodes and retransmit it to other stations, routing protocol. Examples of well-known WSNs routing protocols: LEACH (Low-Energy Adaptive Clustering Hierarchy) [10], PEGASIS (Power-Efficient Gathering in Sensor Information Systems)

[14], TEEN (Threshold sensitive Energy Efficient sensor Network protocol) [12], SEP (Stable Election Protocol for clustered heterogeneous wireless sensor networks) [11], DEEC (Distributed Energy Efficient Clustering algorithm for heterogeneous wireless sensor networks) [1] and APTEEN (Adaptive Periodic Threshold-sensitive Energy Efficient sensor network protocol) [18]. A few of them have taken power efficiency and network lifetime into consideration.

WSN's routing protocols are classified into single-hop and multi-hop routing protocols [24]. In single-hop routing protocols, each node sends its data directly to the BS. In multi-hop routing protocols, nodes send data to intermediate/nearest nodes which similarly forward data to the 
nodes that can directly send the data to the BS. The clustering approach has been designed based on multi-hop technique. In clustering technique, a network is partitioned into groups of sensor nodes called clusters. Each cluster consists of member nodes one of them is designated as a cluster head $(\mathrm{CH})$. The $\mathrm{CH}$ gathers the data from its cluster's members. Then, the CHs transmit the gathered

data to the BS. Dividing the WSN into a huge number of clusters can adversely affect the network efficiency and decreases its lifetime $[15,23]$. In this work, we pay a great attention to $\mathrm{LEACH}$ which is an example of cluster-based routing protocols.

The paper is organized as follow: Section 2 related work, section 3 proposed model, and section 4 simulation and results.

\section{RELATED WORK}

To enhance the WSNs lifetime, several routing protocols are introduced. These routing protocols [23] have drawbacks in terms of the expected network lifetime. Consequently, premature death of the nodes compromises the routing protocols performance.

LEACH algorithm [10] is one of the clustering-based routing algorithms that improves WSNs lifetime. The partition of the WSN into clusters is accomplished by the network, since the nature of the WSN imposes a self-organizing discipline.

To improve the LEACH protocol, Hong et.al [3] present a variant version of LEACH protocol called Threshold- LEACH or TLAECH. T-LEACH limits the number of selected CHs by comparing a candidate $\mathrm{CH}$ residual energy with a threshold. Although they assume that decreasing the number of $\mathrm{CHs}$ increases the WSNs lifetime, the presence of a threshold sometimes obstructs the election of new $\mathrm{CHs}$, reduces the energy levels at the nodes and adversely affects the network lifetime. Arumugam and Ponnuchamy [4] present an energyefficient LEACH (EE-LEACH) protocol for efficient data gathering. The energy-efficient routing is attained by nodes which have the superior residual energy. These superior nodes work as source nodes to the $\mathrm{CHs}$, i.e., assistant nodes. The source nodes are chosen to forward the data to the BS. The source nodes assist to achieve better packet delivery ratio with lower use of energy. Arumugam and Ponnuchamys experimental work shows that EELEACH outperforms the existing LEACH. In a turn, we believe that the source nodes will be depleted at a higher rate for the sake of efficient gathering. Therefore, EE-LEACH may compromise the WSN's lifetime. Agarwal, Kumar, and Prakash [25] introduce ACO-LEACH algorithm that optimizes the path of data transmission between the nodes and the CHs in LEACH. ACO-LEACH improves the performance of LEACH by boosting the nodes and therefore increasing the lifetime of the network.

The idea of having deputies for the cluster heads has been adopted by Ahlawat and Malik [20]and Aziz et. al. [7]. The approach presents a modified version of LEACH protocol called VLEACH, which aims to increase network lifetime. The vice cluster head (VCH) is

a node that assume $\mathrm{CHs}$ responsibility when $\mathrm{CH}$ is absent (dead).

The selection of $\mathrm{VCH}$ has three bases: minimum distance, maximum residual energy, and minimum energy. The shortcoming of the VLEACH approach lies in the overhead imposed by electing new VCHs. Mendis, Guru and Halgamuge [19] propose a mobility feature to the sink. Though, they do not necessitate a topology to the WSN. The mobile sink job is to collect the data from nodes. The main problem of this technique is the difficulty to find a trajectory that enables the collection of data from all nodes as the model has no hierarchy approach. Nayak and Devullapalli [5] propose an enhancement to the LEACH protocol. They call it LEACH with

Fuzzy Descriptors. The aim is to prolong the WSN lifetime. The proposed protocol depends on Fuzzy inference engine (Mamdanis rule) which elect a super cluster head (SCH) from CHs to transfer the gathered data to the mobile BS. The selection of SCH utilizes fuzzy rules and depends on three parameters: the energy level of each $\mathrm{CH}$, mobility and the distance between the 
$\mathrm{CH}$ and the mobile BS. The proposed protocol employs fuzzy descriptors that results in a $20 \%$ overall enhancement to the WSN lifetime compared to LEACH.

Also, we compare our proposed protocol to that of Nayak and Devullapalli [5]. The simulation results show that our protocol outperforms Nayak and Devulapalli's protocol in terms of networ lifetime. MA-LEACH yields a 50\% enhancement in network lifetime compared to $20 \%$ enhancement of LEACH with fuzzy descriptors.

In this work, we introduce a model to enhance the performance of the LEACH protocol. We propose the use of a mobile aggregator (MA). The MA is designed to collect the data from the clusters during the sojourn time. The MA trajectory is optimized using particle swarm optimization. The model has been constrained to fit the physical process as shown in next section. The trip of the MA is optimized by Particle Swarm Optimization (PSO) [9]. Though, the resulting path must meet our model's constraints. PSO is an optimization technique introduced to solve problems for which we do not have polynomial time algorithms [22], so far. We have adapted a solution to the famous Travel Sales Person (TSP) [13] using PSO and constrained it to fit our model's requirements.

In our model, the contributions are two folds: Firstly, we adopt a hierarchical protocol, LEACH in contrast to the work of $\mathrm{Gu}$ et al. [16,] and we do not mobilize the sink as in [2]. Secondly, we limit the number of nodes a mobile aggregator needs to visit to a smaller

set of CHs. A small number of nodes, $\mathrm{CHs}$, makes it computationally feasible to find a trajectory for the mobile aggregator.

\section{PSO AND OPTIMAL TRAJECTORY}

As an evolutionary algorithm, PSO is based on a swarm of particles. The swarm consists of $t$ particles that represent candidate solutions. The particles search the n-dimensional hyperspace looking for the global solution where $\mathrm{n}$ denotes the number of optimal parameters to be determined. A particle i occupies position $X_{i j}$ and velocity $V_{i j}$ in the $j^{\text {th }}$ dimension of hyperspace, $1 \leq \mathrm{i} \leq \mathrm{s}$ and $1 \leq \mathrm{j} \leq \mathrm{n}$. However, an objective function $\mathrm{f}\left(x_{1} ; x_{2} ;::: ; x_{n}\right)$, where $\mathrm{f}: \mathbb{R}^{n} \quad \mathbb{R}$ is used to evaluate each particle

$$
\begin{aligned}
& v_{i j}(t+1)=k_{1} v_{i j}(t)+k_{2}\left(\text { best }_{i j}-x_{i j}(t)\right)+k_{3}\left(\text { gbest }_{i d}-x_{i d}(t)\right) \\
& x_{i j}(t+1)=x_{i j}(t)+v_{i j}(t+1) \\
& \text { best }_{i j}(t+1)=\text { best }_{i j}(t)+v_{i j}(t)
\end{aligned}
$$

Definition 1. Given $\mathrm{N}$ cluster heads $\left\{c h_{1}, c h_{2}, \ldots, c h_{n}\right\}$, the distance between every two CHs $c h_{i, j}$ denoted by $\mathrm{d}\left(c h_{i}, c h_{j}\right)$, the MA has to find a permutation $\mathrm{x}=\left(x_{1}, x_{2}, \ldots, x_{n}\right)$ such that $x_{i} \in\{1,2, \ldots, \mathrm{N}\}$ to minimize the tour length according to the constraints given in Equation 4.

$$
\begin{gathered}
\text { Minimize } \quad \mathrm{L}(\mathrm{x})=\sum_{i}^{N-1} \mathrm{~d}\left(c h_{x_{i}}, c h_{x_{i+1}}\right)+\mathrm{d}\left(c h_{x_{n}}, c h_{x_{1}}\right) \\
\forall_{i} \in\{1,2, \ldots \mathrm{N}\}, \exists_{j} \in\{1,2, \ldots \mathrm{N}\} \\
\mathrm{d}\left(c h_{i}, c h_{j}\right)=\mathrm{d}\left(c h_{j}, c h_{i}\right) \\
r_{t} \geq t r_{t}+s_{t},
\end{gathered}
$$

where $r_{t}, t r_{t}$ and $s_{t}$ are the round time, trip time and sojourn time respectively. 


$$
t r_{t}=\sum_{i}^{N-1} \frac{\mathrm{d}\left(c h_{x_{i}}, c h_{x_{i+1}}\right)}{v}+\frac{\mathrm{d}\left(c h_{x_{n^{\prime}}} c h_{x_{1}}\right)}{v}
$$

\subsection{MA-LEACH Energy Model}

MA-LEACH is an enhancement to the LEACH protocol. LEACH adapts the energy model of Heizelman, Chandrakasan and Balakrishnan [10], see Equation 6.

$$
E_{C H_{i}}=l E_{\text {elec }}\left(\frac{N}{K}-1\right)+l E_{D A} \frac{N}{K}+l E_{\text {elec }}+l \epsilon_{m p} d_{\text {to } B S}^{4}
$$

where $E_{R X}=l E_{\text {elec }}, l$ equals number of bits, $\left(l E_{\text {elec }}+l \epsilon_{m p} d_{\text {to BS }}^{4}\right)$ is the amount of energy dissipation due to transmission (per packet).

The following analysis aims at estimating the amount of save in energy consumption by MALEACH. Consider $r_{t}$ as the collective time CHs work during a particular round, then the use of MA diminishes that time by the sojourn time $s_{t}$. Let $E_{C_{C H}}\left(r_{t}\right)$ be the amount of consumed energy by cluster head i. Then the amount of depleted energy by a cluster-head during the time interval $r_{t}-\frac{s_{t}}{n}$, where $\mathrm{n}$ is the number of $\mathrm{CHs}$ during the given round, is computed by Equation7.

$$
E_{C h_{i}\left(r_{t}-\frac{s_{t_{i}}}{n}\right)}=\left(1-\frac{s_{t_{i}}}{n * r_{t}}\right) E_{c h_{i}\left(r_{t}\right)}
$$

Equation 8 shows that the save in energy, $E_{S}\left(c h_{i}\right)$, is the difference between the consumption without MA and that with the MA.

$$
E_{S}\left(c h_{i}\right)=E_{C h_{i}\left(r_{t}\right)}-E_{C h_{i}\left(r_{t}-\frac{s_{t_{i}}}{n}\right)}
$$

Therefore, the total energy save during the lifetime of the network, $E_{S}$, is computed by Equation 9

$$
E_{S}=\frac{1}{n} \sum_{j=1}^{r} \sum_{i=1}^{n} E_{C h_{i}\left(r_{t}\right)} * s_{t_{i}}
$$

\section{SIMULATION AND RESULTS}

To evaluate the performance of MA-LEACH, we build a software simulation. The simulation experiments are run on a MATLAB program with a setting of a network consists of 100 homogeneous nodes, each with initial energy of 0.5 Joule. The nodes are deployed randomly within a $100 \times 100 \mathrm{~m}$ sensor field. The Base Station (BS) is located at center of the network area, i.e., $(50,50) \mathrm{m}$ (see Table 1). The energy consumption due to communication is calculated using the first order energy model [10]. We assume that each sensor node generates one data packet per time unit transmits it to $\mathrm{CH}$.

Fig. 1 shows a sample run of the LEACH protocol with the aforementioned network settings. Fig. 1 (a) indicates the number of dead nodes versus the number of rounds. It also shows that the first node dies at round number 80 and by the passage of 169 rounds almost more than 90 per cent of the nodes are dead (see Table 2). Fig. 1 (b) gives a relation between the residual energy in nodes and the number of rounds. A sudden/sharp decline in the energy of the nodes takes place from the early beginning of the running of the model, i.e., round number 1 to round number 100, which causes the residual energy to fall to almost 5 (J) (see Table 3).

To test the enhancement provided by the MA-LEACH, we run our simulation against the same network settings. Fig. 2 shows the result of a sample run of the MA-LEACH protocol. Fig. 2 indicates the number of dead nodes versus the number of rounds. It also shows that the first node to die happened at the round number 187 compared to 80 - the round at which the first node dies in LEACH and 95 for Fuzzy-LEACH. By the passage of 210 rounds almost 50 per cent of the nodes are alive compared with 115 rounds for the LEACH protocol and 117 for the FuzzyLEACH. Fig. 2 gives a relation between the residual energy in nodes and the number of rounds. The decline rate of the residual energy in MA-LEACH protocol is less than that of the LEACH 
protocol and LEACH with fuzzy descriptor. In MA-LEACH, it takes 200 rounds to fall below 5 (J) residual energy compared with 110 rounds to the LEACH and 111 rounds to LEACH with fuzzy descriptors. Table 2 and 3 show comparisons among LEACH, LEACH with fuzzy descriptors and MA-LEACH.

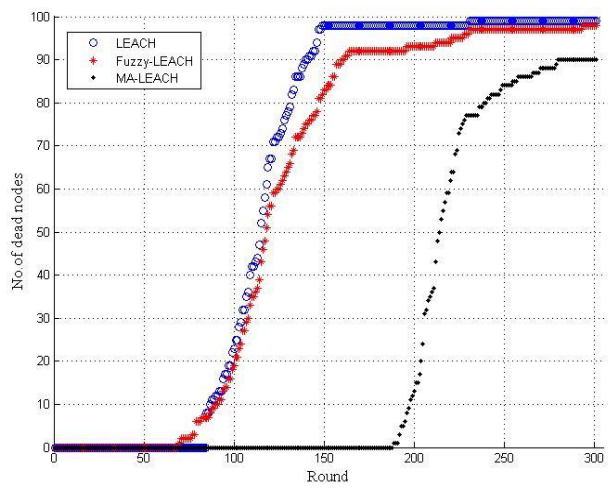

(a)

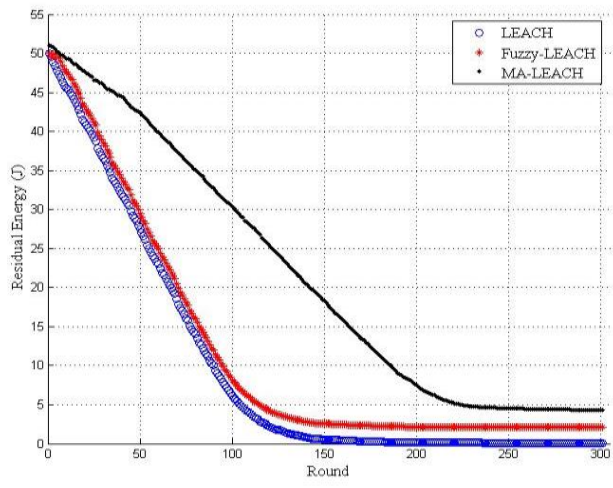

(b)

\section{MA-LEACH}

Fig. 1: Output of a sample run of LEACH and Fuzzy-LEACH versus

a Number of dead nodes versus number of rounds

b Residual energy versus rounds

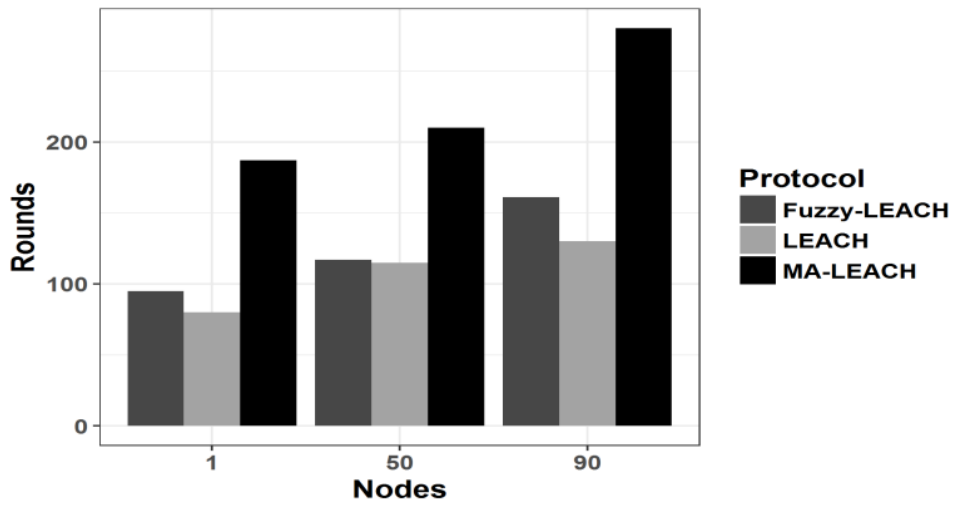

Fig. 2: Performance comparison among LEACH, Fuzzy LEACH and MA-LEACH

Table 1: Simulation parameters

\begin{tabular}{|l|c|}
\hline Parameter & value \\
\hline Network field dimension & $100 \times 100$ \\
\hline Number of Nodes & 100 \\
\hline Nodes deployment & Random \\
\hline BS Location & at $(\mathrm{x}, \mathrm{y})=(50,50)$ \\
\hline Initial energy & $0.5 \mathrm{~J}$ \\
\hline Energy dissipation to operate radio device & $50 \mathrm{NJ} / \mathrm{bit}$ \\
\hline Transmit and receive energy & $50 \mathrm{NJ} / \mathrm{bit}$ \\
\hline Data aggregator energy & $5 \mathrm{NJ} / \mathrm{bit}$ \\
\hline Packet length & $500 \mathrm{bytes}$ \\
\hline Simulation time & $500 \mathrm{sec}$. \\
\hline
\end{tabular}


MA-LEACH: ENERGY EFFICIENT ROUTING PROTOCOL FOR WSNS USING PARTICLE SWARM OPTIMIZATION AND MOBILE AGGREGATOR

Table 2: Nodes Mortality Rate

\begin{tabular}{|l|c|c|c|}
\hline \multirow{2}{*}{ Nodes Mortality } & \multicolumn{3}{|c|}{ Round no. } \\
\cline { 2 - 4 } & LEACH & Fuzzy-LEACH & MA-LEACH \\
\hline First node & 80 & 96 & 187 \\
\hline Last $10 \%$ alive nodes & 169 & 203 & 239 \\
\hline Last 5\% alive nodes & 190 & 228 & 247 \\
\hline
\end{tabular}

Table 3: Residual Energy and Mortality

\begin{tabular}{|c|c|c|c|c|}
\hline & \multicolumn{2}{|c|}{ Residual Energy in (J) } & \multicolumn{2}{c|}{ Nodes Mortality } \\
\hline Round no. & 150 & 200 & 150 & 200 \\
\hline LEACH & 2 & 1 & 85 & 95 \\
\hline Fuzzy-LEACH & 4 & 3 & 87 & 97 \\
\hline MA-LEACH & 15 & 3 & $<5$ & 10 \\
\hline
\end{tabular}

\section{CONCLUSION AND FUTURE WORK}

In this work, we introduce an enhancement to the LEACH protocol. The proposed protocol outperforms the LEACH in terms of network lifetime and energy utilization within the nodes. The introduced MA alleviates the load on the $\mathrm{CHs}$ as to which we attribute the improvement measured by the simulation. The trajectory of the MA is determined by utilizing swarm intelligence (PSO) and is enforced to meet the constraints of the network. Further improvements could be conducted in future work, such as testing the effect of multiple MAs or adding assumption on CHs. For example, we may study the effect of using superior CHs with rechargeable capabilities, and test the WSN performance accordingly.

\section{REFERENCES}

[1] Intanagonwiwat, C., Govindan, R., Estrin, D., Heidemann, J., and Silva, F.: 'Directed diffusion for wireless sensor networking', IEEE/ACM Transactions on Networking, 2003, 11, (1), pp. 2-16

[2] Bi, Y.., Sun, L., Ma, J., et al. :HUMS: An autonomous moving strategy for mobile sinks in data-gathering sensor networks', EURASIP Journal on Wireless Communications and Networking, 2007, 2007, (5), url=http://dx.doi.org/10.1155/2007/64574

[3] Hong, J., Kook, J., Lee, S., and et al. :'T-LEACH: The method of threshold-based cluster head replacement for wireless sensor networks', Inf. Sys. Front., 2008, 11, (5), pp. 513-521

[4] Arumugam, G.., and Ponnuchamy, T. :"EE-LEACH: development of energy-efficient LEACH Protocol for data gathering in WSN', EURASIP Journal on Wireless Communications and Networking, 2015, (1), doi:"10.1186/s13638-015-0306-5"

[5] Nayak, P., and Devulapalli, A.:'A Fuzzy Logic-Based Clustering Algorithm for WSN to Extend the Network Lifetime', IEEE Sensors Journal, 2016, 16(1), pp. 137-144

[6] Arora, V.K., Sharma, V. and Sachdeva, M.: 'A survey on LEACH and others routing protocols in wireless sensor network', Optik-International Journal for Light and Electron Optics, Aug.2016, 127(16), pp.6590-6600

[7] Aziz, L., Raghay, S., Aznaoui, H., and Jamali, A.: 'A new enhanced version of VLEACH protocol using a smart path selection', International Journal of GEOMATE, 2017, 12(30), 28-34

[8] Parrado-Garcia, F.J., Vales-Alonso, J., and Alcaraz, J.: 'Optimal planning of WSN deployments for in situ lunar surveys', IEEE Transactions on Aerospace and Electronic Systems, 2017, PP(99) 
[8] Parrado-Garcia, F.J., Vales-Alonso, J., and Alcaraz, J.: 'Optimal planning of WSN deployments for in situ lunar surveys', IEEE Transactions on Aerospace and Electronic Systems, 2017, PP(99)

[9] Kennedy , J., and Eberhart, R.: 'Particle swarm optimization'. Proc. IEEE Int. Conf. Neural Networks, NJ, USA, Jan. 1995, pp. 1942-1948

[10] Heinzelman, W., Chandrakasan, A., and Balakrishnan, H.: 'Energy-efficient communication protocol for wireless microsensor networks'. IEEE Hawaii Int. Conf. System Sciences, Cambridge,MA, USA, Jan. 2000, pp. 10-20

[11] Intanagonwiwat, C., Govindan, R. and Estrin, D.: 'Directed diffusion: a scalable and robust communication paradigm for sensor networks', In: Sixth Annual International Conference on Mobile Computing and Networking, 6(11), Aug. 2000, Boston, MA, USA, pp. 56-67

[12] Chakrabarti, A., Chandrakasan, A., Sabharwal, A., and et al.: 'Using predictable observer mobility for power efficient design of sensor networks'. Proc. 2nd Int. Conf. Inf. Processing in Sensor Networks, Palo Alto, CA, USA, Apr. 2003, pp. 129-145

[13] Wang, K., Chandrakasan, Huang, L., Zhou, C., and et al. : 'Particle swarm optimization for traveling salesman problem'. Proc. Int. Conf. Machine Learning and Cybernetics , Washington, DC, USA, Nov. 2003, pp. 1583-1585

[14] Somasundara, A., Romamoorthy, A., and Srivastava, M.: 'Mobile element scheduling for efficient data collection in wireless sensor networks with dynamic deadlines'. IEEE Int. RealTime Systems Symposium, Los Angeles, CA, USA, Dec. 2004, pp. 296-305

[15] Luo, J., and Hubaux , J.: 'Joint mobility and routing for life-time elongation in wireless sensor networks'. 24th Annual Joint Conference of the IEEE Computer and Communications Societies, Lausanne, Switzerland, March 2005, pp. 1735-1746

[16] Gu, Y., Bozdag, D., Ekici, E., and et al. : 'Partitioning based mobile element scheduling in wireless sensor networks'. In: Proc. IEEE SECON, Santa Clara, CA, USA, Sept. 2005, pp. 386395

[17] Guru, S., Halgamuge, S., and Fernando, S.: 'Particle swarm optimisers for cluster formation in wireless sensor networks'. Int. Conf. Intelligent Sensors, Sensor Networks and Information Processing, Melbourne, Australia, Dec. 2005, pp. 319-324

[18] Ma, M., Chandrakasan, A., and Yang, Y.: 'Clustering and load balancing in hybrid sensor networks with mobile cluster heads'. Int.1 Conf. Quality of Service in Heterogeneous Wired/Wireless Networks, Waterloo, Canada, Aug. 2006, pp. 7-9

[19] Mendis, C., Guru, S., and Halgamuge, S.: 'Optimized sink node path using particle swarm optimization'. 20th International Conference on Advanced Information Networking and Applications- Volume 1 (AINA'06), Vienna, Austria, Apr. 2006.

[20] Ahlawat, A., and Malik, V.: 'An extended vice-cluster selection approach to improve V LEAH protocol in WSN'. IEEE 3rd Conf. Advanced Computing and Communication Technologies (ACCT), April 2013, pp. 236-240

[21] Begum, K., and DixitInt, K.:'Industrial WSN using IoT: A survey', Con. on Electrical, Electronics, and Optimization Techniques (ICEEOT), March, 2016, pp. 499-504

[22] Papadimitriou, C. and Steiglitz, K.: 'Combinatorial optimization: algorithms and complexity'. (Prentice-Hall, Inc., 1982)

[23] Boukerche, A., Araujo, R. and Silva, F. :'A context interpretation based wireless sensor network for the emergency preparedness class of applications', in Nikoletseas, S. (Ed.): 'Algorithmic aspects of wireless sensor networks' (Springer, 2006), pp. 25-34

[24] Frey, H., Ruhrup, S. and Stojmenovic, I.: 'Guide to wireless sensor networks' (Springer, 2009)

[25] Agarwal, T., Kumar, D., Prakash, N.: 'Prolonging network life time using ant colony optimization algorithm on LEACH protocol for wireless sensor networks' in Meghanathan, N., Boumerdassi, S., and Chaki, N., and et al. (Ed.): 'Recent trends in networks and communications' (Springer, 2010) pp. 634-641 